Supporting information for

\title{
Chameleon-Inspired Strain-Accommodating Smart Skin
}

Yixiao Dong, ${ }^{\dagger}$ Alisina Bazrafshan,${ }^{\dagger}$ Anastassia Pokutta,,${ }^{\ddagger}$ Fatiesa Sulejmani, ${ }^{\ddagger}$ Wei

Sun, ${ }^{\ddagger}$ J. Dale Combs ${ }^{\dagger}$ Kimberly C. Clarke, ${ }^{\dagger}$ and Khalid Salaita ${ }^{*} \dagger$

$\dagger$ Department of Chemistry, Emory University, Atlanta, Georgia 30322, United States.

¥Wallace H. Coulter Department of Biomedical Engineering, Georgia Institute of Technology, Atlanta, Georgia 30332, United States. 


\section{Table of Contents}

Supplementary S1: Estimation of strain in conventional responsive PC gels.............3

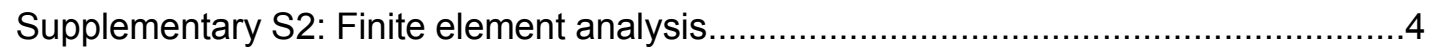

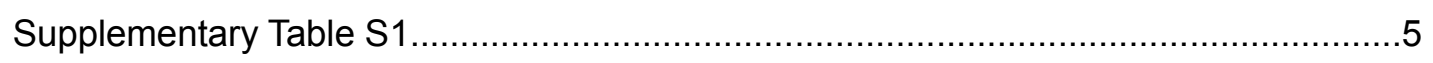

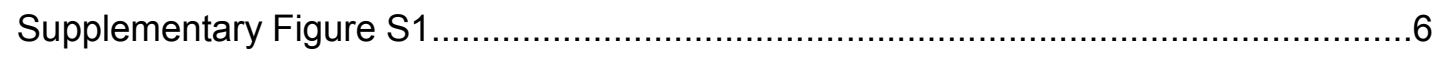

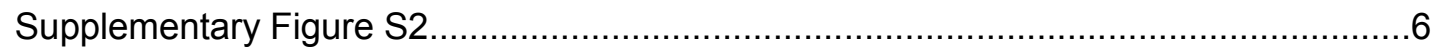

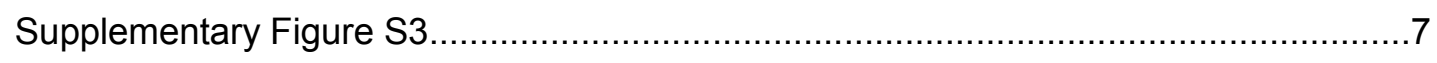

Supplementary Figure S4

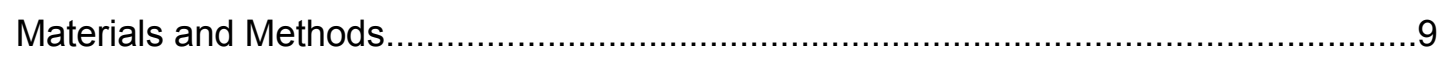

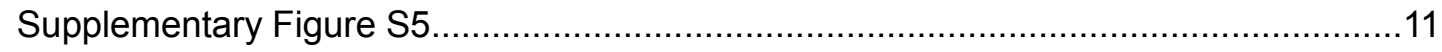

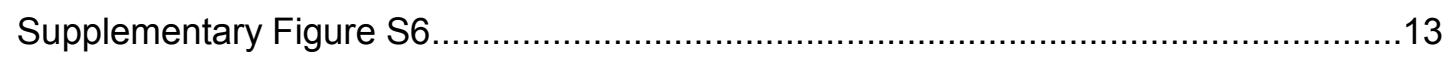

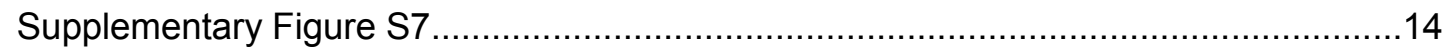

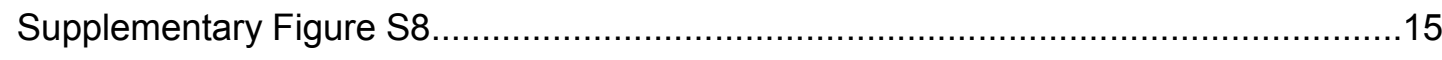

Supplementary S3: Deformation of SASS materials ..............................................16

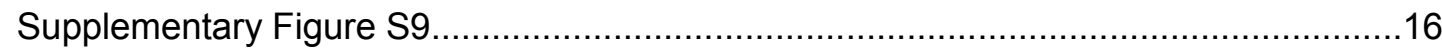

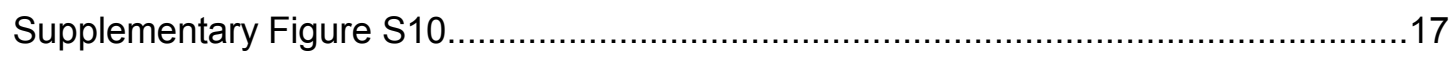

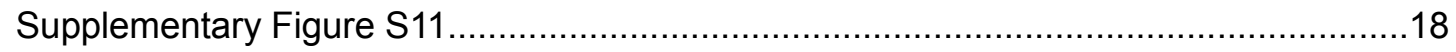

Supplementary S4: Calculation of photothermal efficiency of MNPs...................19

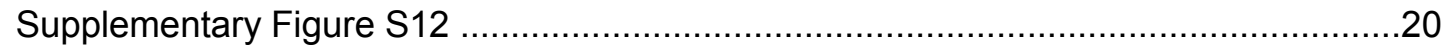

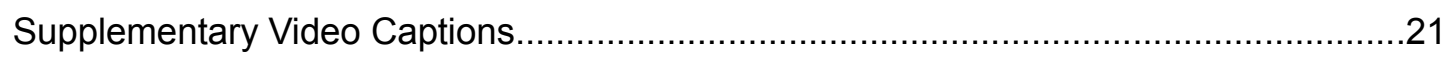

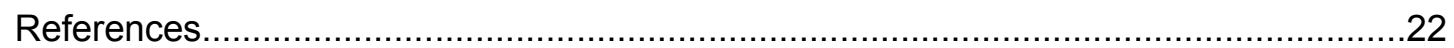




\section{Supplementary S1: Estimation of strain in conventional responsive photonic crystal gels}

The diffraction of RPC gels can be estimated by the following equation, ${ }^{1,2}$

$$
m \lambda=2 n d \sin \theta
$$

(equation S1)

where $\lambda$ is the diffraction wavelength, $d$ is the lattice spacing, $\theta$ is the angle of incidence, $m$ is the diffraction order (considering the first order diffraction, $m=1$ ), $n$ is the overall refractive index of the photonic crystal gels. According to previous studies, ${ }^{3} n$ can be calculated as the average refractive index as below,

$$
n_{\text {avg }}=\phi n_{\text {particle }}+(1-\phi) n_{\text {matrix }} \quad \text { (equation S2) }
$$

where $\phi$ is the volume fraction of the particle in RPC gel, $n_{\text {particle }}$ is the refractive index of nanoparticles that form the photonic crystal structure. $n_{\text {matrix }}$ is the refractive index of the hydrogel matrix (pNIPAM in this work). In our work, the content of nanoparticles in RPC gels is below $0.5 \mathrm{wt} . \%$, and therefore, $n_{\text {avg }} \approx n_{\text {matrix. }}$. Also, the change in refractive index ${ }^{4}$ as a result of the phase transition of pNIPAM is less than $1 \mathrm{wt} \%$, thus we can treat the refractive index as a constant value here. We use $n_{0}$ to symbolize the refractive index of pNIPAM hydrogel. The final equation can be rewritten as,

$$
\lambda=2 n_{0} d \sin \theta \quad \text { (equation S3) }
$$

In this equation, the diffraction wavelength is proportional to the lattice distance.

1) If $\theta=90^{\circ}$, then $\sin \theta=1, d=\lambda / 2 n_{0}$. For a sample with an original diffractive wavelength of $500 \mathrm{~nm}$, and a blue shift of $\Delta \lambda=100 \mathrm{~nm}$, the overall change in $d$ is as large as $20 \%$. In other words, the strain in the sample is $20 \%$.

2) If $\theta<90^{\circ}$, then $\sin \theta<1, d=\lambda / 2 n_{0} \sin \theta(d>\lambda / 2)$. In the same case as of 1$)$, the deswelling ratio must be larger than $20 \%$ strain. The results are $23.1 \%$ if $\theta=60^{\circ}$ and $40 \%$ if $\theta=30^{\circ}$.

These calculations explain the inevitable large-scale swelling/deswelling behavior of traditional RPC gels. Note that this calculation represents a lower bound estimation of the required strain because we ignore the volume of the nanoparticles comprising the PC. Because the particles are incompressible, the actual required deswelling of the hydrogel must be longer than our current estimation. 


\section{Supplementary S2: Finite element analysis}

A thermal stress model was applied for all the mechanical calculations used in this work. ${ }^{5}$ First, thermal strains were calculated according to the equation below,

$$
\varepsilon_{\text {th }}=\alpha(T)\left(T-T_{\text {ref }}\right)
$$

Where $\varepsilon_{\text {th }}$ is the strain of thermal expansion. $\alpha(T)$ is the coefficient of thermal expansion (linear). This coefficient was set as -0.01 to simulate the deswelling of pNIPAM. The temperature jump used for these calculations ( $T$ - $\left.T_{r e f}\right)$ was set to $50 \mathrm{~K}$, which justifies the assumed linear shrinkage of $50 \%$.

Next, the solid mechanics were calculated based on the series of equations indicated below:

$$
\begin{array}{cr}
0=\nabla \cdot \sigma & \text { (equation S5) } \\
\varepsilon_{\mathrm{el}}=s: \sigma & \text { (equation S6) } \\
\varepsilon=\frac{1}{2}\left[(\nabla u)^{\mathrm{T}}+\nabla u\right] & \text { (equation S7) } \\
\varepsilon=\varepsilon_{\mathrm{el}}+\varepsilon_{\mathrm{th}} & \text { (equation S8) }
\end{array}
$$

The parameters used in equations 5-8 are detailed in Table $\mathbf{S 1}$ below. 
Table S1. Parameters used for solid mechanics calculations

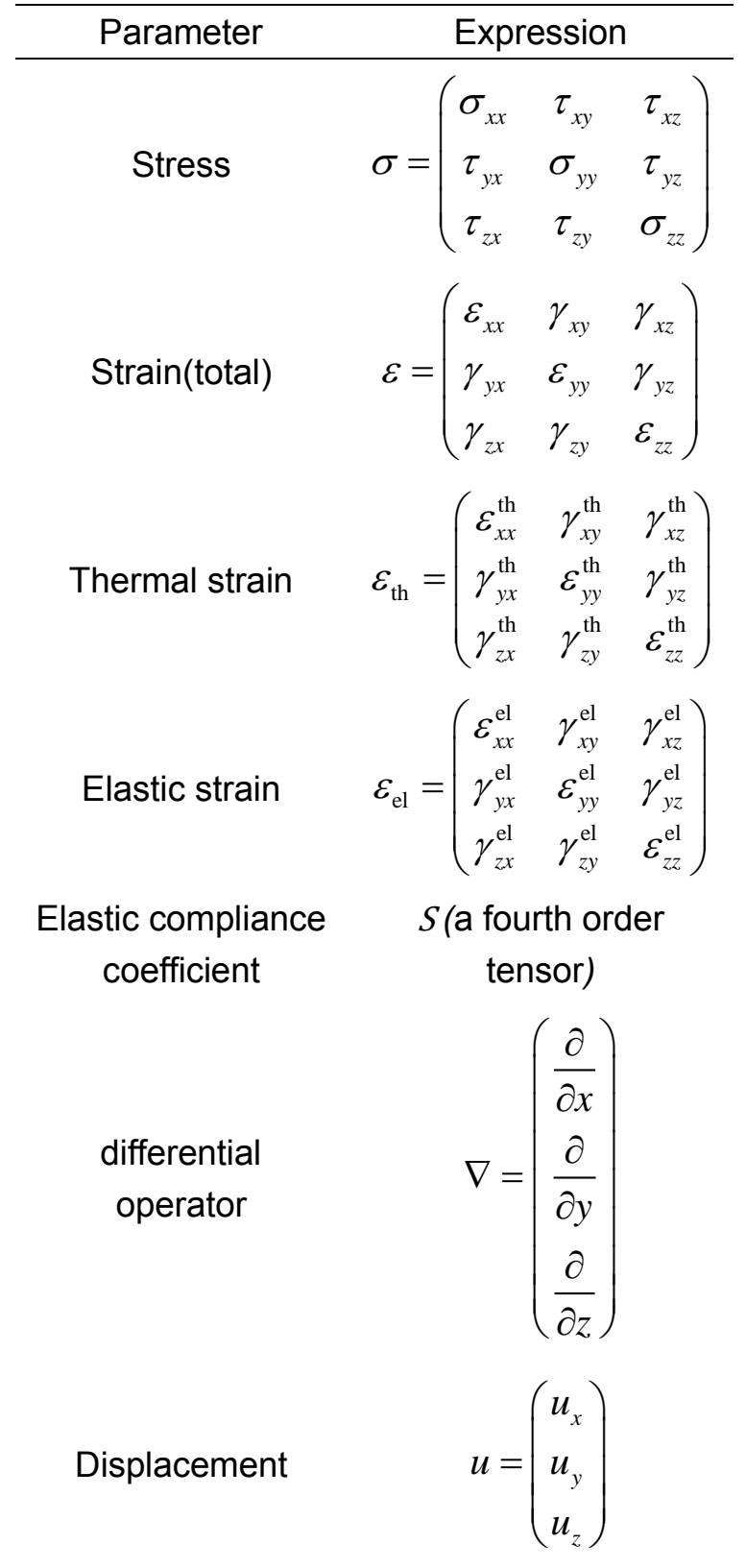



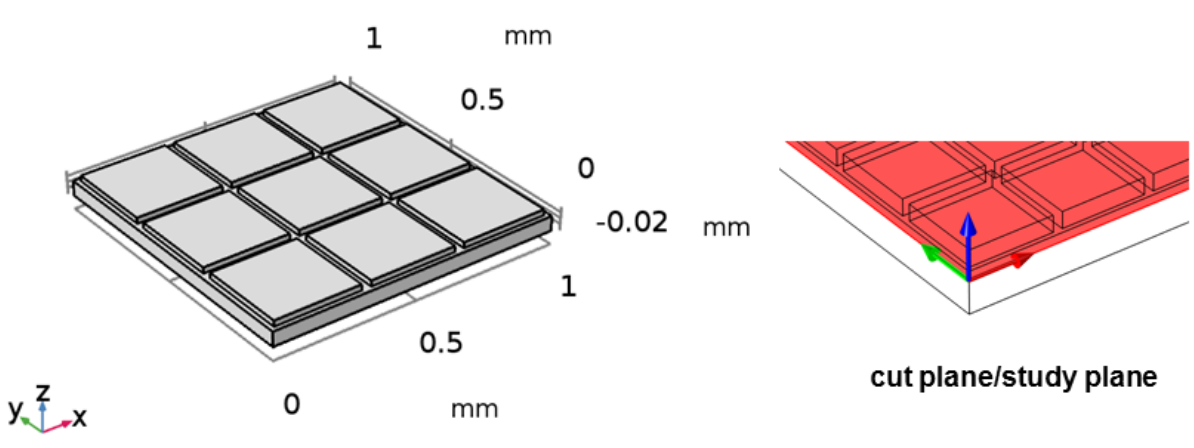

cut plane/study plane

Figure S1. The model build for the finite element analysis calculations with a top-layer array of $3 \times 3$ (left) and the chosen cut plane for deformation and force calculations (right).

\begin{abstract}
A typical model built for finite element analysis is show in Figure S3. The dimensions of the bottom layer were $1 \times 1 \times 0.05 \mathrm{~mm}$. The number (Num) of top-layer squares in a row can vary from 1 to 9 . To guarantee that the overall area of the top layer is constant, the side length of each square was defined as $0.9 \mathrm{~mm} / \mathrm{Num}$. Note that the dimensions of the model are smaller than those used for the experimental samples; however, we were focused on the relative trends as predicted by the computational analysis. Thus, the specific dimensions used in the calculations are not critical for our conclusions.
\end{abstract}




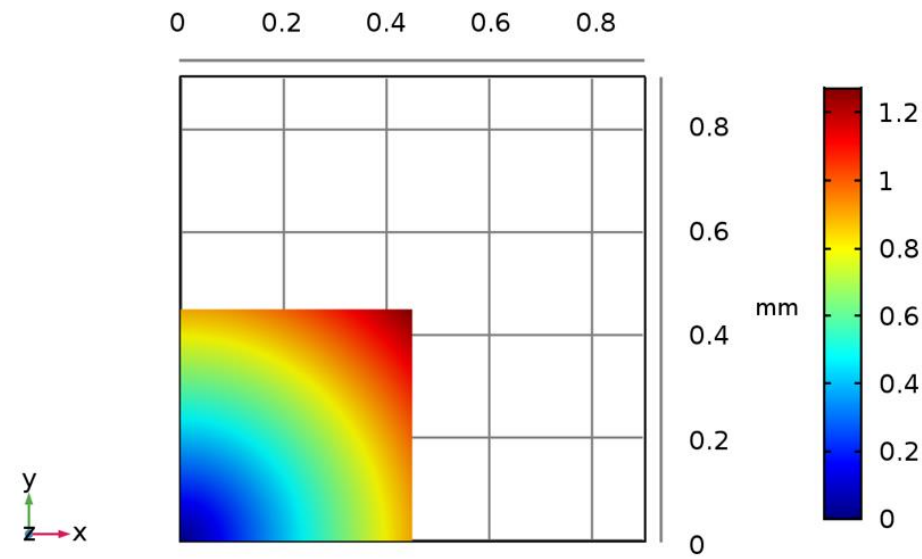

Figure S2. Plot of total displacement of a responsive (pNIPAM) film. For this calculation, we assumed a $50 \%$ linear shrinkage of the responsive layer that was suspended without a supporting polymer layer. One corner of the structure was fixed $(x, y, z)=(0,0,0)$, and thus the structure shrank towards the fixed point. The colored heat map indicates the total displacement in units of $\mathrm{mm}$.

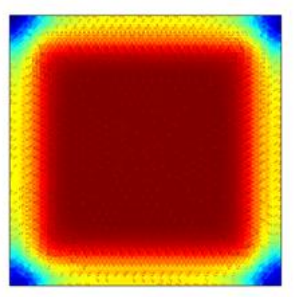

$1 \times 1$

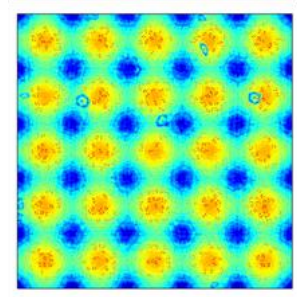

$5 \times 5$

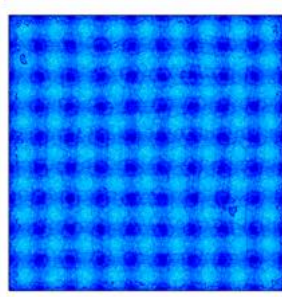

$9 \times 9$

Figure S3. Images represent plots of von Mises stress for SASS structures that have been patterned with specific densities of square arrays. The Von mises stress values were obtained from a specific cut plane (see Figure S2).

von Mises stress analysis of a chosen cut plane further revealed a relationship between the number of tiles that a SASS material was divided to, and the Von mises stress (Figure S4). We found that fabricating SASS films with more finely patterned square arrays leads to a decrease in the accumulated strain. This observation can be partially explained because the patterned arrays spread the stress over a greater area as the structure is more finely patterned. In other words, the split structure provides more interstitial "space" between the responsive PC elements to dissipate and spread the accumulated stress.

We used one-dimensional strain analysis to further reveal the strain-accommodating behavior of SASS (Figure S4). The displacement field in Figure S4(b) shows staircaseshaped plots across distance (Arc length) of the red line depicted in Figure S4(a). The plot 
demonstrates that increasing the number of squares in a row (from $1 \times 1$ to $9 \times 9$ ), leads to more frequent "steps" across the same distance, which decreases the total displacement of the material. Each step corresponds to the space between the responsive polymer squares. In other words, the elastic deformation of the interstitial space dampens the total displacement so that the overall material is less deformed (Figure S4 (b) and (c)). Therefore, fabricating higher density square arrays (miniaturizing the patterns) in the responsive top layer increases the strain capacitance of the supporting material. These calculations provide a quantitative prediction of the strain-accommodating behavior for SASS.

a

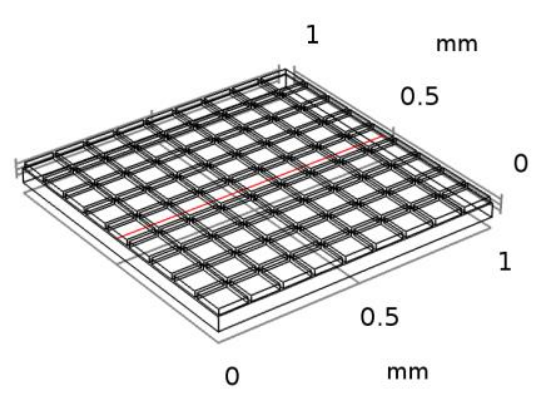

b

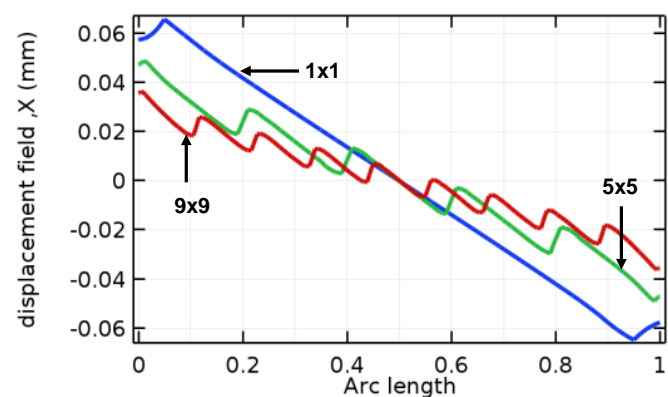

C

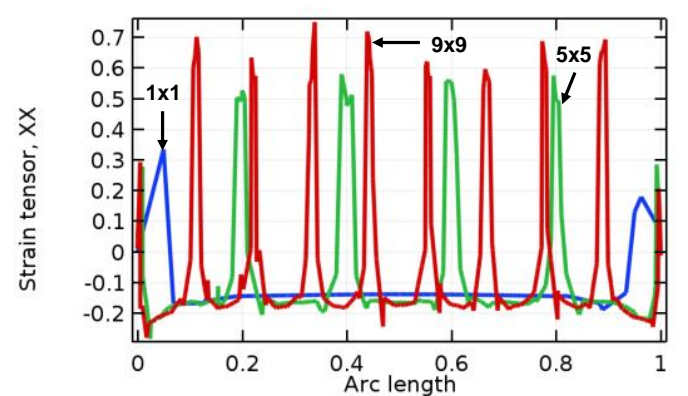

Figure S4. Computational prediction of strain values across the $x$ direction of SASS films. a. 3D wire mesh rendering of SASS denoting the location of the line scan (red line, $x$ direction) to quantify the displacement. b. Plot of displacement field (deformation) across the selected line (red line) for SASS materials patterned with differing numbers of tiles. The blue, green, and red plots denote SASS films with $1 \times 1,5 \times 5$, and $9 \times 9$ arrays, respectively. c. First derivative of displacement field (strain tensor) from plot in $\mathbf{b}$, which shows the strain capacitance of the interstitial spaces between the responsive squares. Note that in this model, we fixed the center of the bottom of the supporting layer. This is why the plot shown in $\mathbf{b}$ had negative values. In other words, the whole material deforms toward the center, where a positive value represents a deformation toward the positive $x$-axis direction, and negative values indicate deformation in the opposite direction. 


\section{Materials and Methods}

Materials. Sodium pyrophosphate decahydrate $\left(\mathrm{Na}_{4} \mathrm{P}_{2} \mathrm{O}_{7} \cdot 10 \mathrm{H}_{2} \mathrm{O}, \geq 99 \%\right)$, anhydrous ferric chloride $\left(\mathrm{FeCl}_{3}, \geq 97 \%\right.$ ), ethylene glycol ( $\mathrm{EG}, \geq 99 \%$ ), sodium acetate (NaAc, $\geq 99 \%$ ), sodium hydroxide $(\mathrm{NaOH}, \geq 99 \%$ ), tetraethoxysilane (TEOS, $\geq 99 \%)$, ammonium hydroxide $\left(\mathrm{NH}_{3} \cdot \mathrm{H}_{2} \mathrm{O}, 25 \% \sim 28 \%\right)$, Poly(4-styrenesulfonic acid-co-maleic acid) sodium salt (PSSMA, $\mathrm{Mw} \sim 20 \mathrm{k}, \mathrm{SS}: M A=1: 1$ ), D-isoascorbic acid (D-iAA,), N-isopropylacrylamide (NIPAM, $\geq 99 \%$ ), 2,2-dimethoxy-2-phenylacetophenone (Irgacure, $\geq 99 \%$ ), poly(ethylene glycol) diacrylate (PEGDA, Mn 700), and (-)-riboflavin ( $\geq 98 \%$ ) were purchased from Sigma-Aldrich (St. Louis, MO). Laponite XLG was generously provided by BYK Additive Inc. (Louisville, KY). Anhydrous ethanol was purchased from Decon Labs Inc. (King of Prussia, PA). Amine terminated tetra-polyethylene oxide (4arm-PEG-NH,$M w \sim 10 k$ ) was purchased from Laysan Bio Inc. (Arab, AL). Pentaerythritol tetra(succinimidyloxyglutaryl) polyoxyethylene (tetra-PEG-NHS, Mw 10k) was purchased from NOF America Corporation (White Plains, NY). Sylgard $(184$ and its curing agent were purchased from Dow Corning Corporation (Midland, MI). Deionized (DI) water was purified using a Barnstead Nanopure water system (18.2 $\mathrm{M} \Omega$ resistivity). Delrin ${ }^{\circledR}$ (Polyoxymethylene Resin) sheets were obtained from McMaster-Carr (Douglasville, GA).

Fabrication of SASS. An overview of the process for preparing SASS films is illustrated in Figure 1f, and is comprised of several steps that are detailed below. Briefly, the first step involves the synthesis of magnetic nanoparticles (step A). These are then aligned and crosslinked within a Delrin $®$ mold (step $B$ ) to create a patterned responsive photonic crystal film (step C). The responsive photonic crystal was subsequently cut using a patterned Delrin $®$ grid. Next, the supporting polymer film was poured over the responsive photonic crystal array. Finally, the structure was allowed to cure to generate the SASS film (step D).

A. Synthesis of $\mathrm{Fe}_{3} \mathrm{O}_{4} @ \mathrm{SiO}_{2}$ core-shell nanoparticles (MNPs). $\mathrm{Fe}_{3} \mathrm{O}_{4} @ \mathrm{SiO}_{2}$ coreshell nanoparticles were synthesized according to previously developed methods. ${ }^{6}$ Briefly, for particles with an average diameter of $180 \mathrm{~nm}, 0.65 \mathrm{~g} \mathrm{FeCl}_{3}, 40 \mathrm{~mL} \mathrm{EG}, 3.0$ g NaAc, 1.05 g PSSMA, $14 \mathrm{mg} \mathrm{D-iAA}$, and $120 \mu \mathrm{L} \mathrm{DI} \mathrm{H}_{2} \mathrm{O}$ were added consecutively into a parafilm sealed beaker. After vigorous magnetic stirring for about $40 \mathrm{~min}$, a homogeneous mixture formed and then $0.6 \mathrm{~g} \mathrm{NaOH}$ was added. The mixture was stirred for 1-2 $\mathrm{h}$ until all the $\mathrm{NaOH}$ was completely dissolved. The mixture was then transferred into a capped $50 \mathrm{~mL}$ Erlenmeyer flask and then allowed to react in a preheated oven $\left(190{ }^{\circ} \mathrm{C}\right.$ ) for $9 \mathrm{~h}$. The $\mathrm{Fe}_{3} \mathrm{O}_{4}$ products were separated from the solution using an external rare-earth (neodymium-iron-boron) magnet and washed. Initially, the particles were washed with $30 \mathrm{~mL}$ of $50 \mathrm{v} / \mathrm{v} \%$ ethanol three times, and then washed three additional times with DI water.

After the washing steps described above, the magnetite particles were re-dispersed in $30 \mathrm{~mL}$ of DI water. A $12 \mathrm{~mL}$ aliquot of this dispersion was then mixed with $80 \mathrm{~mL}$ ethanol and $4 \mathrm{~mL}$ ammonium hydroxide (25-28 wt\%) under vigorous mechanical stirring for 1 
min. This solution was warmed by using a water bath at $50{ }^{\circ} \mathrm{C}$. Subsequently, two aliquots of $0.4 \mathrm{~mL}$ TEOS were added every $20 \mathrm{~min}$. Finally, the reaction products were washed with ethanol three times by using magnetic separation for each wash.

To generate particles with an average size of $210 \mathrm{~nm}$, we followed the steps described above with two exceptions. First, the amount of $\mathrm{H}_{2} \mathrm{O}$ used was decreased to $105 \mu \mathrm{L}$. Second, the TEOS was added in three aliquots (rather than two): $0.4,0.4$, and $0.2 \mathrm{~mL}$ at 20 min intervals.

B. Synthesis of pure silica nanoparticles. Silica nanoparticles $(\sim 300 \mathrm{~nm})$ were synthesized by the well-established stober method. ${ }^{7} 50 \mathrm{~mL}$ ammonium solution was made by mixing $9 \mathrm{~mL}$ ammonium hydroxide (25-28 wt\%), $16 \mathrm{~mL}$ ethanol, and $25 \mathrm{~mL}$ D.I. $\mathrm{H}_{2} \mathrm{O}$. This solution was swiftly added to $50 \mathrm{~mL} 0.4 \mathrm{M}$ TEOS ethanol solution under vigorous stirring. After reacting for $2 \mathrm{~h}$, the particles were centrifuged and washed three times with ethanol to collect monodisperse silica nanoparticles. TEM indicated that the average particle size was approximately $300 \mathrm{~nm}$.

C. Fabrication of Delrin $®$ mold and gel cutting tools. Square shaped sheets of Delrin $®$ with a thickness of 0.125 inch $(0.3175 \mathrm{~cm})$ were cut into the desired shapes using a laser cutter (Universal Laser Systems VLS 4.60, featuring a 60 -watt $\mathrm{CO}_{2}$ laser). The patterns were designed using the AutoCAD software package.

D. Synthesis of responsive photonic film. The as-obtained $\mathrm{Fe}_{3} \mathrm{O}_{4} @ \mathrm{SiO}_{2}$ particles from the protocol (described above) were redispersed in $12 \mathrm{~mL}$ of ethanol, and then $3 \mathrm{~mL}$ of this solution of $\mathrm{Fe}_{3} \mathrm{O}_{4} @ \mathrm{SiO}_{2}$ was redispersed in $\mathrm{EG}$ solution containing $8 \mathrm{mg}$ riboflavin, followed by adding $0.09 \mathrm{~g}$ Irgacure, $225 \mu \mathrm{L}$ PEGDA, and $0.45 \mathrm{~g}$ NIPAM. The mixture was vortexed for $10 \mathrm{~s}$ to obtain a homogeneous solution that was then sonicated for $5 \mathrm{~min}$ in the dark. We describe this mixture as the "precursor solution" in subsequent steps. A $3 \mathrm{~cm} \times 3 \mathrm{~cm}$ Delrin mold covered with a piece of transparent petri dish was used to fabricate the responsive photonic crystal layer. For each synthesis, $350 \mu \mathrm{L}$ precursor solution was injected into the mold and exposed to a magnetic field of approximately 245 Gauss (determined using a WT10A teslameter). Finally, the precursor solution was cured with a $100 \mathrm{~W}$ high intensity UV lamp (365 nm, Analytik Jena US company) for $10 \mathrm{~min}$. The as-obtained thin film was washed with DMF and $\mathrm{H}_{2} \mathrm{O}$ three times, respectively, then was stored in water for further use. Notably, for special shapes (fish and leaf), the Delrin $®$ molds were deliberately fabricated into the required shapes by using a laser cutter as described above using AutoCAD to draw the design.

E. Patterning of SASS. The as-synthesized responsive photonic film was first cut by firmly pressing a patterned Delrin $®$ grid (Figure S5, fabricated by a laser cutter, as noted above). This was performed on a clean petri dish surface. Prior to the preparation of the PEG supporting layer, two precursor solutions were prepared. The first (precursor A) was prepared using $750 \mu \mathrm{L}$ deionized water and $0.03 \mathrm{~g}$ Laponite XLG 
that were mixed together in a $2 \mathrm{~mL}$ vial using a magnetic stir bar until the solution became clear. To this Laponite XLG solution, $750 \mu \mathrm{L}$ of $0.1 \mathrm{M} \mathrm{Na}_{4} \mathrm{P}_{2} \mathrm{O}_{7} / \mathrm{H}_{3} \mathrm{PO}_{4}$ buffer $(\mathrm{pH}=7.2)$ and $0.12 \mathrm{~g}$ tetra-PEG-NH $\mathrm{N}_{2}$ were added and completely dissolved through vigorous stirring and sonication. The second precursor solution $(\mathrm{B})$ was prepared using $0.12 \mathrm{~g}$ tetra-PEG-NHS that was dissolved in $600 \mu \mathrm{L} \mathrm{Na}{ }_{4} \mathrm{P}_{2} \mathrm{O}_{7} / \mathrm{H}_{3} \mathrm{PO}_{4}$ buffer $(\mathrm{pH}=7.2)$ under vigorous stirring and sonication. Both precursors $\mathrm{A}$ and $\mathrm{B}$ were stored in an ice box for $20 \mathrm{~min}$ before mixing in order to slow the gelation rate. After cooling precursors $A$ and $B$, the solutions were mixed together for $10 \mathrm{~s}$ using a magnetic stir bar, then quickly poured into a specific Delrin $®$ mold (Figure S5) and sandwiched with the gridcut photonic film that was placed on a petri dish. The gelation process was complete within $15 \mathrm{~min}$ at room temperature.
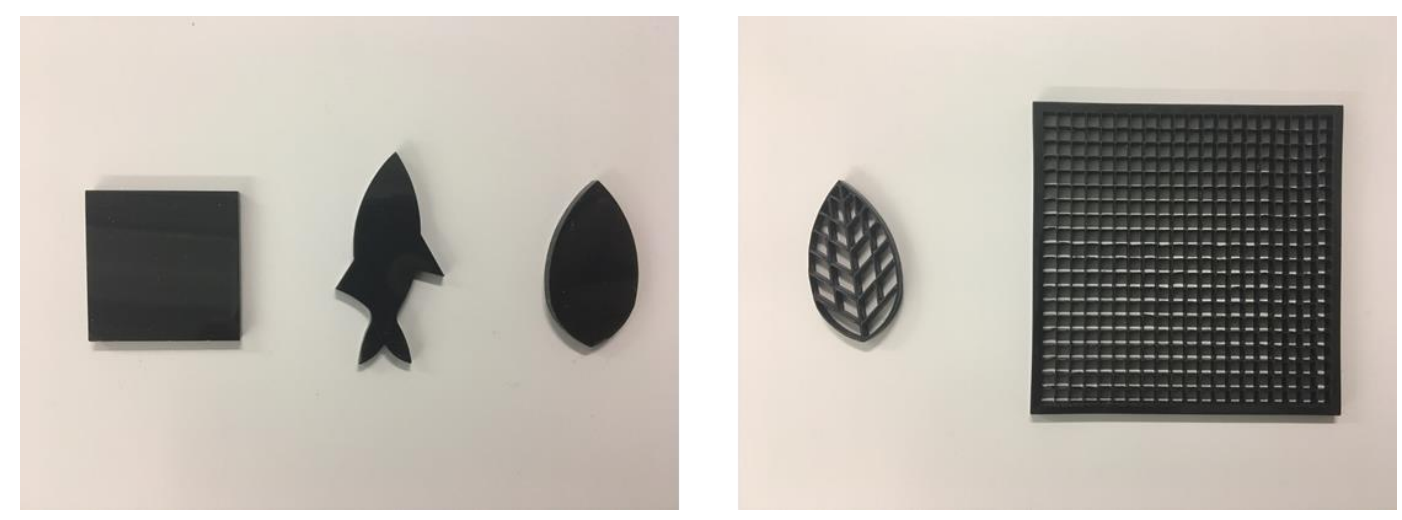

Figure S5. Photographs of Delrin $®$ mold (left) and Delrin ${ }^{\circledR}$ gel cutting grids (right) used in this paper.

Characterization. TEM images were acquired with an Hitachi HT-7700 with $80 \mathrm{kV}$ accelerating voltage. SEM images were obtained using a Topcon DS-130F Field Emission SEM. Digital photographs and videos were recorded with an iPhone 6s. Dynamic surface temperature change was monitored by an Etekcity Lasergrip 1080 Non-Contact Digital Laser Infrared Thermometer Temperature Gun (instrument temperature range is $-50{ }^{\circ} \mathrm{C}$ to $550{ }^{\circ} \mathrm{C}$ ). Dynamic light scattering (DLS) was performed with a NanoPlus Zeta / Nano Particle Analyzer at $25^{\circ} \mathrm{C}$. UV light intensity was determined by AMTAST UVA365 UV light meter. Gel deswelling ratio was measured by a digital caliper. White LED illumination was performed using a Cygolite Metro 800 with light intensity of 800 lumen (distance to the sample: $3 \mathrm{~cm}$ ).

Solar responses were tested with natural sunlight during the daytime between the hours

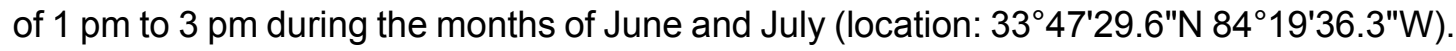
All experiments were conducted indoors at constant temperature of approximately $23^{\circ} \mathrm{C}$ with air conditioning. Note that the sunlight passed through a glass window and a PDMS film before reaching the SASS samples. Accordingly, the actual sunlight power density may be up to $20 \%$ lower than the outdoor solar density due to the absorption of PDMS and glass, and the interfacial reflection.

Laser response test were conducted with a 50-80 mW $532 \mathrm{~nm}$ Green Laser Module with 
TTL and Fan (12V) purchased from OdicForce Lasers (Surbiton, UK). The equipment set is described in Figure 5a. Note that the SASS sample was totally immersed in water while the laser stimulation experiments were conducted.

Reflectivity spectra were measured with a FLAME-S-VIS-NIR Spectrometer equipped with a premium $400 \mu \mathrm{m}$ reflection probe (Ocean Optics Inc., Dunedin, FL). The spectrometer was calibrated with a diffuse reflectance standard (PTFE) prior to all the measurements. All the spectra data were recorded through the OceanView 1.6.3 software package.

Uniaxial tensile tests were conducted using a TestResources 100Q Universal Testing Machine (Shakopee, MN). Samples were mechanically loaded at a rate of $5 \mathrm{~mm} / \mathrm{min}$ until failure. A CCD camera was used to capture optical marker motion, while an in-house LabVIEW (National Instruments, Austin, TX, USA) program was used to synchronize marker locations and load information with each video frame. Young's moduli were quantified as the slope of the stress-strain curve.

All optical imaging was performed using a Nikon Eclipse Ti microscope, operated with Nikon Elements software, a 1.49 NA CFI Apo 100x objective, perfect focus system, LED light source along with a laser launch excitation source. An RICM filter cube with a $535 \mathrm{~nm}$ excitation filter was employed to visualize the particle chains. To image the polymer autofluorescence we used a quad filter cube set (Chroma TRF89902-EM - ET 405/488/561/647 $\mathrm{nm}$ ) while exciting the sample using a TIRF $405 \mathrm{~nm}$ excitation launch with $80 \mathrm{~mW}$ power. ImageJ was used to analyze the intensity change of the time lapse video. 

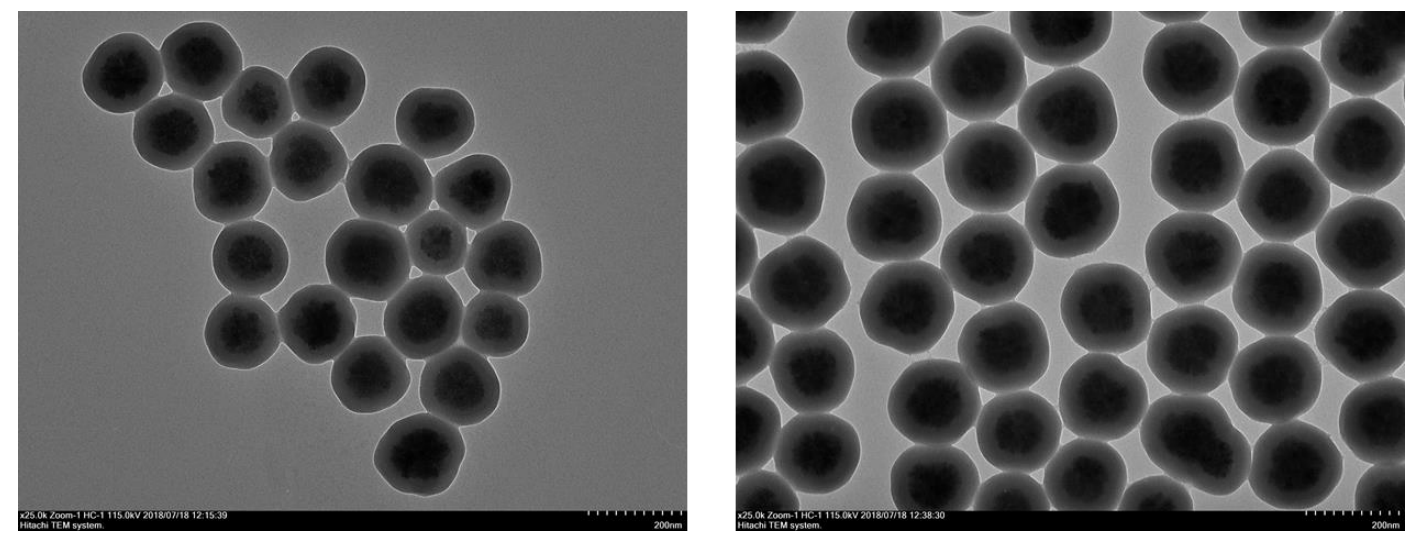

Figure S6. TEM images of $180 \mathrm{~nm}$ (left) and $210 \mathrm{~nm}$ (right) $\mathrm{Fe}_{3} \mathrm{O}_{4} @ \mathrm{SiO}_{2}$ nanoparticles. The diameters were determined by calculating the average diameter of over 100 particles in TEM images (scale bar $200 \mathrm{~nm}$ ). 


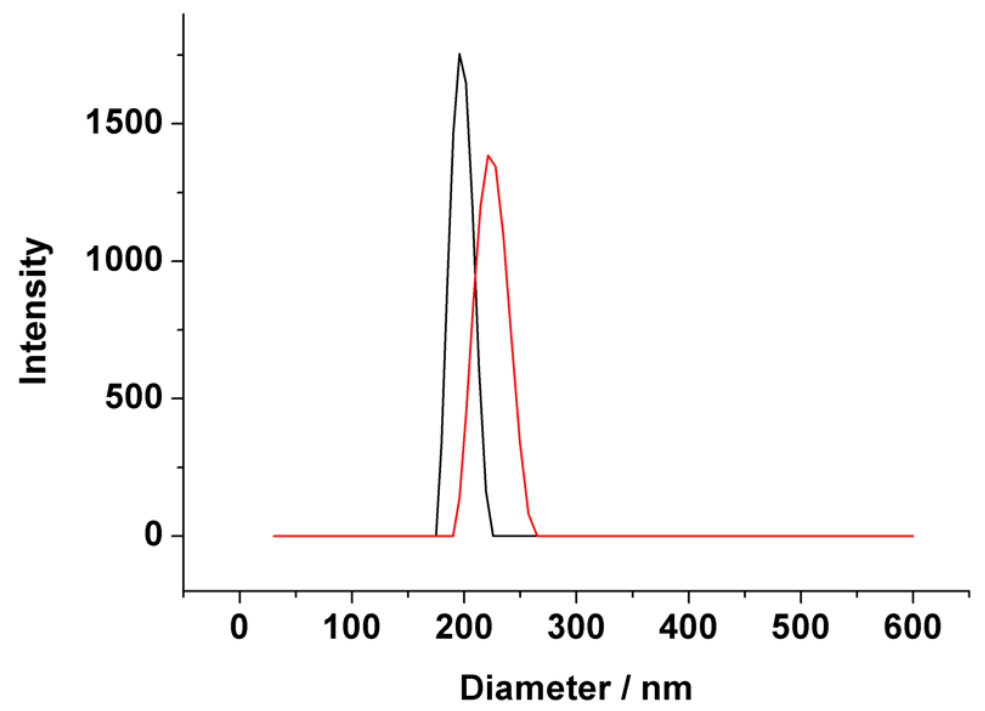

Figure S7. Representative dynamic light scattering (DLS) spectra of two different sizes of particles. The average hydrodynamic diameter of the smaller particles was $196 \pm$ $3.1 \mathrm{~nm}$ which is consistent with the TEM determine size of $180 \mathrm{~nm}$. The large particles displayed diameter of $223 \pm 4.7 \mathrm{~nm}$ and is consistent with the $210 \mathrm{~nm}$ diameter determined from TEM. Note that DLS results on average size of particles is greater than the TEM measurement due to the solvent layer surrounding the particles. The reported DLS diameters were averaged from five measurements. 


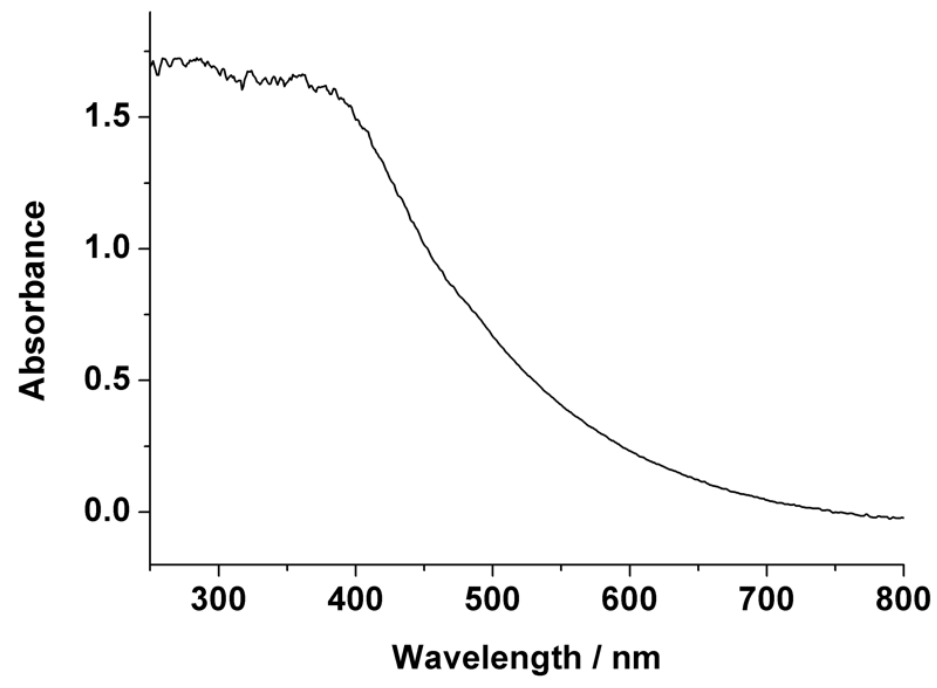

Figure S8. A UV-vis absorbance spectrum of a diluted $\mathrm{Fe}_{3} \mathrm{O}_{4} @ \mathrm{SiO}_{2}$ dispersion ( 0.001 $w t \%)$. The particles have strong absorbance in the UV region that drops down in the visible, in agreement with previous reports. ${ }^{1}$ 


\section{Supplementary S3: Deformation of SASS materials}

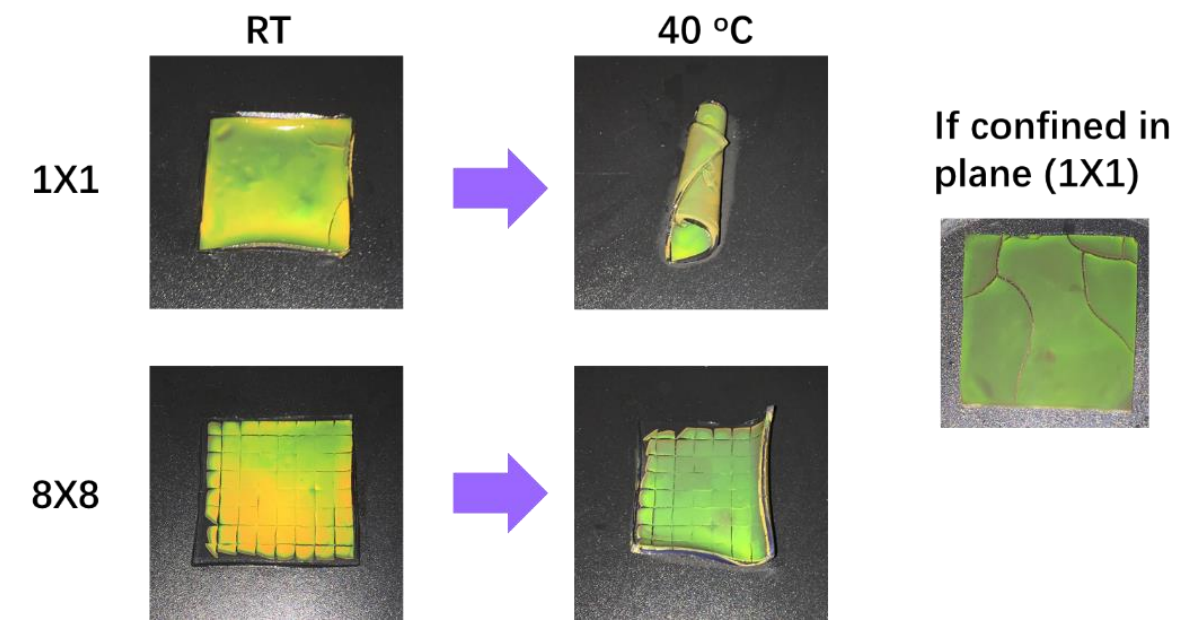

Figure S9. Photographs comparing deformation of SASS $1 \times 1$ and $8 \times 8$ films. The films were about $3.4 \times 3.4 \mathrm{~cm}$ at room temperature. The samples were heated by using a water bath and were removed from the water in order to acquire the photographs.

As further evidence supporting the importance of the segmented structure for reducing the deformation of SASS materials, we also include photographs depicting the deformation of free-standing SASS films with differing segmentation patterns (Figure S9). Unlike the results presented in Figure $\mathbf{2} e$ and $\mathbf{f}$ in the main text, these SASS materials were allowed to bend freely without any restriction. Based on the images, the deformation of $1 \times 1$ segmentation is more significant compared to that of the $8 \times 8$ segmented SASS, validating the experimental results presented in Figure 1d. Interestingly, due to the low mechanical strength of pNIPAM responsive PC gels, the $1 \times 1$ segmented SASS cracks and breaks apart because it was sandwiched and forced to remain in a 2D plane (photograph to the right of Figure S9). The deformation predicted by COMSOL in the main text (Figure 1d) was not observed in these experiments because modeling assumed that both layers are unbreakable. Nevertheless, we found that the $8 \times 8$ segmented structure is less likely to crack due to the diminished deformation and low average forces on the plane (as shown in Figure S4). 


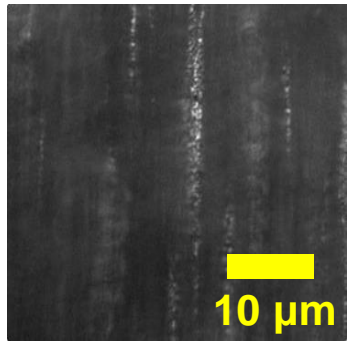

RICM

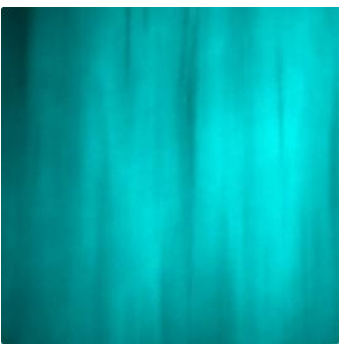

405

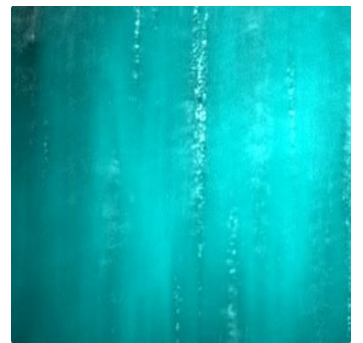

Merge

Figure S10. Microscopy images of a cross-section of responsive layer of SASS observed by different channels and their merged image. Note that the patterns observed by RICM and the $405 \mathrm{~nm}$ channels are highly co-localized. 

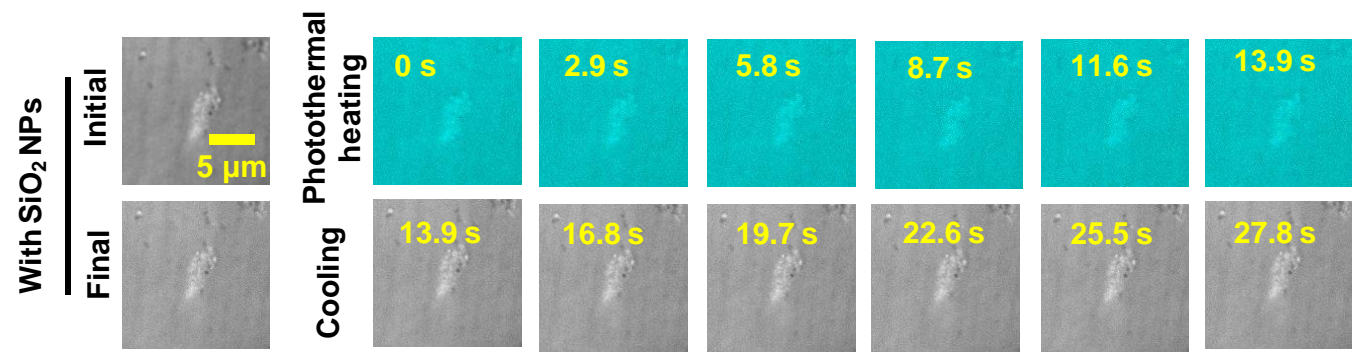

Figure S11. A control experiment of photothermal heating in a SASS sample which the MNPs were replaced with pure silica nanoparticles $(d=300 \mathrm{~nm})$ in the responsive layer. Time lapse images in both photothermal heating and cooling process show no obvious change on particle patterns, which indicates magnetite plays an important role on photo-thermal heating of SASS material. 


\section{Supplementary S4: Calculation of photo-thermal efficiency of MNPs}

The photo thermal efficiency was calculated by the equation set below

$$
\begin{array}{cc}
\sum_{i} C_{i} m_{i} d T / d t=P_{a b}-P_{\text {pass }} & \text { (equation S9) } \\
\eta=P_{a b} / P_{\text {in }} & \text { (equation S10) }
\end{array}
$$

Where $C_{i}$ stands for the heat capacitance of each component inside the cuvette system. mi stands for the mass of each component inside the cuvette system. $P_{a b}$ stands for the power that absorbed by the sample. $P_{\text {pass }}$ stands for the power of light that pass through the sample (not being absorbed). $d T / d t$ is the rate of sample heating. $\mathrm{P}_{\text {in }}$ stands for the power input of light which can be calibrated by a UV light meter. $\eta$ is the photo thermal efficiency.

Because nearly $100 \%$ of MNPs dispersion consists of water and almost all the light passing through the sample is absorbed $\left(P_{\text {pass }} \approx 0\right)$. Equation 9 can be further simplified as

$$
C_{w} m_{w} d T / d t=P_{a b}
$$

(equation S11)

Where $C_{w}$ and $m_{w}$ are the heat capacity of water and the mass of water respectively.

$\mathrm{dT} / \mathrm{dt}$ can be measured through the slope of the tangential line (red dash line) in Figure $3 e(\mathrm{dT} / \mathrm{dt}=0.01013 \mathrm{~K} / \mathrm{s})$. Also, according to UV light meter, $P_{\text {in }}=76.83 \mathrm{~mW} ; \mathrm{C}_{\mathrm{w}}$ and $\mathrm{m}_{\mathrm{w}}$ are known for $4.19 \mathrm{~J} /(\mathrm{g} \cdot \mathrm{K})$ and $1.5 \mathrm{~g}$. As a result, $\eta=4.19^{*} 1.5^{*} 0.01013^{*} 1000 / 76.83 \approx 81.8 \%$. 

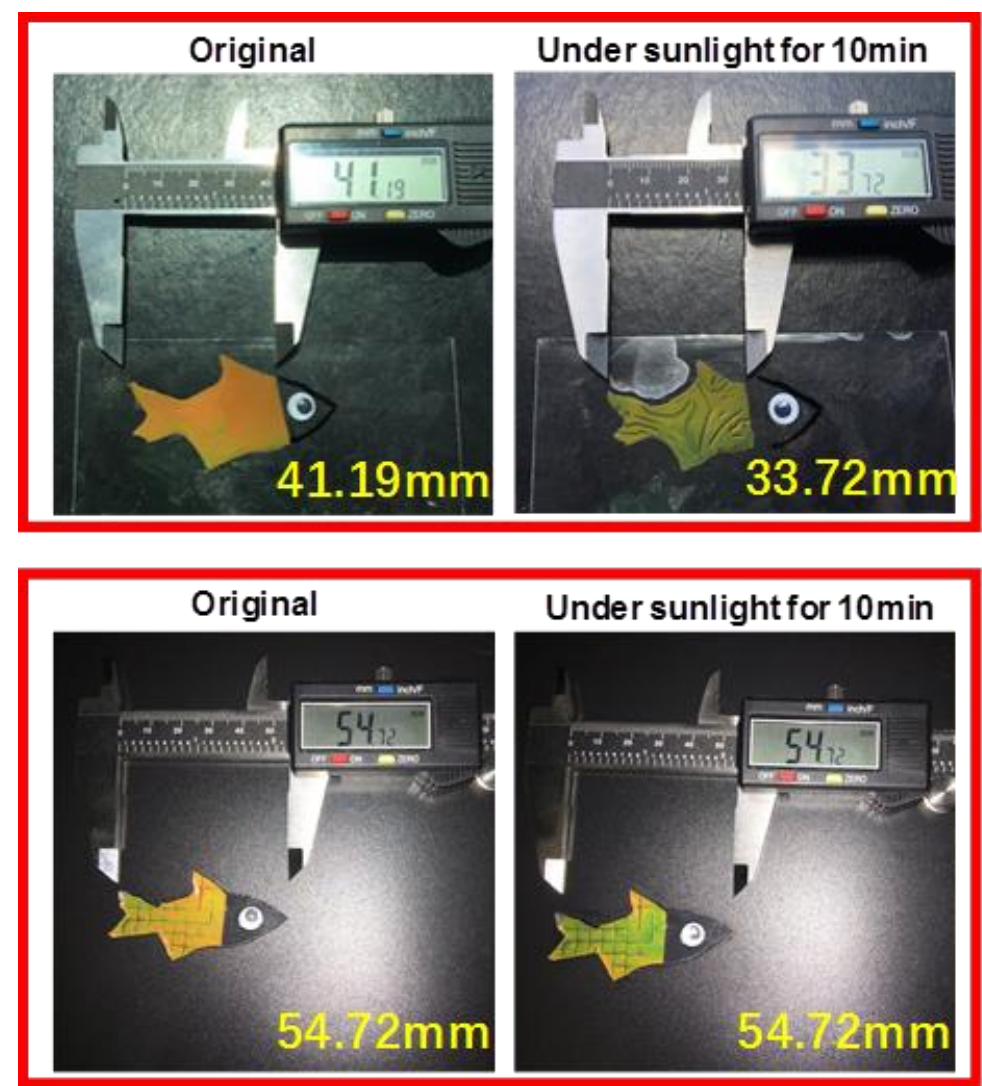

Figure S12. Photographs of fish-shaped RPC and SASS materials before and after exposure to natural sunlight for 10 minutes. The caliper reading is displayed in yellow for clarity and demonstrates the advantage of the SASS design (upper images of traditional responsive PC, and lower images of SASS). 


\section{Supplementary Movie captions:}

Movie S1. Time-lapse video of aligned MNPs $(\mathrm{d}=180 \mathrm{~nm})$ in the responsive layer collected during irradiation with a $405 \mathrm{~nm}$ laser. The video was collected with a 100x oil immersion objective in the $405 \mathrm{~nm}$ channel and has 70 frames in total with $4.83 \mathrm{fps}$. Observed changes in the nanochain pattern are due to photothermal heating since the observation plane is constant. (displayed with $\times 7$ speed)

Movie S2. Time-lapse video of aligned MNPs $(\mathrm{d}=180 \mathrm{~nm})$ in the responsive layer collected during sample cooling after the $405 \mathrm{~nm}$ laser irradiation was stopped. The video was collected with a 100x oil immersion objective in the RICM channel and has 70 frames in total with $4.83 \mathrm{fps}$. Observed changes in the nanochain pattern are due to gel recovery after photo-thermal heating since the observation plane is constant. (displayed with $x 7$ speed)

Movie S3. Time-lapse video of pure silica nanoparticles $(\mathrm{d}=300 \mathrm{~nm})$ in the responsive layer collected during irradiation with a $405 \mathrm{~nm}$ laser. The video was collected in the $405 \mathrm{~nm}$ channel and has 70 frames in total with $4.83 \mathrm{fps}$. (displayed with $x 7$ speed)

Movie S4. Time-lapse video of pure silica nanoparticles $(\mathrm{d}=300 \mathrm{~nm})$ in the responsive layer collected after $405 \mathrm{~nm}$ laser irradiation. The video was collected in RICM channel and has 70 frames in total with $4.83 \mathrm{fps}$. (displayed with $\times 7$ speed)

Movie S5. A typical SASS sample when irradiated with a $532 \mathrm{~nm}$ laser. One square of the array was irradiated for $2 \mathrm{~s}$ followed by $11 \mathrm{~s}$ of cooling down. Upon irradiation with the laser the observed color changes from green to blue due to photothermal heating. After cooling the square returns to its original state. Only the square exposed to the laser light exhibits a color change. Note that the size of each square is $4 \mathrm{~mm} \times 4 \mathrm{~mm}$. 


\section{References}

1. Tang, J.; Myers, M.; Bosnick, K. A.; Brus, L. E., Magnetite Fe3O4 Nanocrystals: Spectroscopic Observation of Aqueous Oxidation Kinetics. J. Phys. Chem. B 2003, 107, 7501-7506.

2. Weissman, J. M.; Sunkara, H. B.; Albert, S. T.; Asher, S. A., Thermally Switchable Periodicities and Diffraction from Mesoscopically Ordered Materials. Science 1996, 274, 959-963.

3. Aguirre, C. I.; Reguera, E.; Stein, A., Tunable Colors in Opals and Inverse Opal Photonic Crystals. Adv. Funct. Mater. 2010, 20, 2565-2578.

4. Philipp, M.; Aleksandrova, R.; Müller, U.; Ostermeyer, M.; Sanctuary, R.; MüllerBuschbaum, P.; Krüger, J. K., Molecular versus Macroscopic Perspective on the Demixing Transition of Aqueous PNIPAM Solutions by Studying the Dual Character of the Refractive Index. Soft Matter 2014, 10, 7297-7305.

5. Sadd, M. H., Elasticity: theory, applications, and numerics. Academic Press: Oxford. 2009; pp 31-53

6. Dong, Y.; Wen, B.; Chen, Y.; Cao, P.; Zhang, C., Autoclave-Free Facile Approach to the Synthesis of Highly Tunable Nanocrystal Clusters for Magnetic Responsive Photonic Crystals. RSC Adv. 2016, 6, 64434-64440.

7. Stöber, W.; Fink, A.; Bohn, E., Controlled Growth of Monodisperse Silica Spheres in the Micron Size Range. J. Colloid Interface Sci. 1968, 26, 62-69. 